ARTICLE

Received 7 Jan 2015 | Accepted 25 Jul 2015 | Published 8 Sep $2015 \quad$ DOl: 10.1038/ncomms9173

\title{
Borromean three-body FRET in frozen Rydberg gases
}

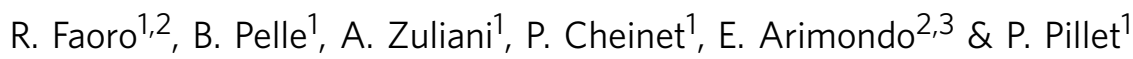

Controlling the interactions between ultracold atoms is crucial for quantum simulation and computation purposes. Highly excited Rydberg atoms are considered in this prospect for their strong and controllable interactions known in the dipole-dipole case to induce non-radiative energy transfers between atom pairs, similarly to fluorescence resonance energy transfer (FRET) in biological systems. Here we predict few-body FRET processes in Rydberg atoms and observe the first three-body resonance energy transfer in cold Rydberg atoms using cold caesium atoms. In these resonances, additional relay atoms carry away an energy excess preventing the two-body resonance, leading thus to a Borromean type of energy transfer. These few-body processes present strong similarities with multistep FRET between chromophores sometimes called donor-bridge-acceptor or superexchange. Most importantly, they generalize to any Rydberg atom and could lead to new implementations of few-body quantum gates or entanglement.

\footnotetext{
${ }^{1}$ Laboratoire Aimé Cotton, CNRS, Univ. Paris-Sud, ENS Cachan, Bât. 505, 91405 Orsay, France. ${ }^{2}$ Physics Department, Universita di Pisa, Largo Pontecorvo 3 I-56127 Pisa, Italy. ${ }^{3}$ INO-CNR, Via G. Moruzzi 1, 56124 Pisa, Italy. Correspondence and requests for materials should be addressed to P.C. (email: patrick.cheinet@u-psud.fr).
} 
U ltracold atom experiments are recognized as promising for quantum simulation ${ }^{1,2}$ and have gathered important achievements, reproducing many-body Hamiltonians as for instance the Superfluid to Mott insulator transition ${ }^{3}$, the Bose-Einstein Condensate to Bardeen-Cooper-Schrieffer (BEC-BCS) crossover ${ }^{4}$ and the Tonks-Girardeau gas ${ }^{5,6}$ or realizing complex one-body Hamiltonian as the quantum transport problem of Anderson localization in three dimensions ${ }^{7,8}$ for which no exact solution is known.

In most of these experiments, the atomic interaction is a key parameter and its control, when possible, is a valuable asset. Highly excited atoms, usually called Rydberg atoms, display strong tunable interactions and have thus been proposed for both quantum simulation ${ }^{9}$ and quantum computation ${ }^{10,11}$ using the interaction blockade ${ }^{12}$ induced by their van der Waals interactions scaling typically as $n^{11}$ with $n$ the principal quantum number. This approach has already proven successful, exploiting the Rydberg blockade ${ }^{13,14}$ for the consecutive creation of entanglement ${ }^{15}$ and implementation of a quantum gate $e^{16}$. More recently, Rydberg-Rydberg interactions have been used to apply a phase shift in an atomic interferometer ${ }^{17}$. These results are demonstrating Rydberg atoms potential.

The van der Waals interactions arise from out-of-resonance dipole-dipole interactions, which can be tuned to resonance under the proper electric field ${ }^{18}$ thanks to the Stark effect. When this condition is fulfilled, they can also lead to non-radiative energy transfers called Förster resonances due to their similarity with fluorescence resonance energy transfer ${ }^{19}$ (FRET) that has been presented by Förster ${ }^{20}$ to explain energy transport in biological systems, after a purely quantum description by Perrin $^{21}$. The theoretical coherent nature of FRET has been recently demonstrated in Rydberg atoms ${ }^{22}$ thanks to the precise control of atomic positions in this experiment.

The similarity between Rydberg physics and FRET in biological systems has motivated the use of Rydberg atoms for the study of quantum energy transport ${ }^{23,24}$ induced by two-body FRET in many-body systems. In parallel, biological studies are trying to determine to what extent the quantum nature of FRET remains at ambient temperature ${ }^{25}$ and how few-body mechanisms might influence the energy transport: multistep FRET $^{26}$ has been demonstrated using relay chromophores or quantum dots, even for non-resonant relays ${ }^{27}$ in so-called donor-bridge-acceptor or superexchange configurations ${ }^{28}$.

Similarly, few-body interactions have recently found a renewed interest in ultracold atomic physics community as paving the way between two-body and many-body physics, as for Efimov physics ${ }^{29,30}$, three-body recombination ${ }^{31}$, macromolecule formation as Rydberg macro-trimers ${ }^{32,33}$ and few-body interactions $^{34}$, with a special interest to the so-called Borromean interactions displaying strong three-body interactions with a negligible contribution of two-body interactions ${ }^{33,35}$. Nevertheless, these studies remained up to now theoretical in Rydberg physics where no Borromean interaction could be demonstrated.

The present work predicts new few-body FRET processes in Rydberg atoms and presents the first observation of a three-body FRET in a cold Rydberg gas. It corresponds to a generalization of the usual two-body FRET where a third atom serves as a relay for the energy transport. This relay also compensates for the energy mismatch preventing the direct two-body FRET between the donor and the acceptor which thus results in a Borromean threebody energy transport. The predicted few-body FRET can be generalized for any quantum system displaying two-body FRET from quasi-degenerate levels. It thus promises important applications in the formation of macro-trimers, implementation of few-body quantum gates, few-body entanglement or heralded entanglement.

\section{Results}

Two-body resonant interaction. In cold Rydberg atoms physics, FRET resonance condition is usually achieved applying an electric field $F$ that Stark shifts the involved energy levels ${ }^{18}$, enabling to find a large set of resonances. In caesium ${ }^{36}$ a well known two-body FRET is described by the equation:

$$
2 \times n P_{3 / 2} \leftrightarrow n S_{1 / 2}+(n+1) S_{1 / 2} .
$$

where $n$ is the principle quantum number, $S$ (respectively $P$ ) represents the orbital angular momentum 0 (resp. 1) of the excited electron and the subscript specifies the total angular momentum $J$. We notice that this resonance exists for both total angular momentum projections $\left|m_{J}\right|=1 / 2$ and $\left|m_{J}\right|=3 / 2$ of an initial $n P_{3 / 2}$ state or for a mixture of both. For convenience, the Rydberg states $n S_{1 / 2}, \quad(n+1) S_{1 / 2}, \quad n P_{3 / 2}\left|m_{J}\right|=1 / 2 \quad$ and $n P_{3 / 2}\left|m_{j}\right|=3 / 2$ will now be labelled as $s, s^{\prime}, p$ and $p^{\prime}$, respectively, with respective energies $E_{s}, E_{s^{\prime}}, E_{p}$ and $E_{p^{\prime}}$ which all depend on the applied electric field $F$. It is important to notice that the zero-field $p$ and $p^{\prime}$ degeneracy is lifted around the resonant FRET electric field, providing a small but finite energy difference that we define as $\delta_{p p^{\prime}}=E_{p^{\prime}}-E_{p}$ on the order of several tens of $\mathrm{MHz}$. As this is much smaller than the energy difference of around $10 \mathrm{GHz}$ between $s, s^{\prime}$ and $p$ or $p^{\prime}$, equation (1) represents an energy transfer between an initial species ( $p$ states) and a different one ( $s$ states).

We introduce the one atom energy mismatch $\Delta$ between the starting state and the average energy of the end state, so-called Förster defect. For the two starting states $p$ and $p^{\prime}$ we define $\Delta_{p}=\frac{E_{s}+E_{s^{\prime}}-2 E_{p}}{2}$ and $\Delta_{p^{\prime}}=\frac{E_{s}+E_{s^{\prime}}-2 E_{p^{\prime}}}{2}$. The different two-body FRET resonances are associated to an initial couple of $p$ states (II label), initial $p^{\prime}$ states (II' label), and finally to an initial $p+p^{\prime}$ couple (III label) with respective resonance conditions $\Delta_{p}=0$, $\Delta_{p^{\prime}}=0$ and $\Delta_{p}=-\Delta_{p^{\prime}}=\delta_{p p^{\prime}} / 2$. The schematic three-body energy diagram of Fig. 1a shows how the three possible two-body FRET resonances arrange as a function of the electric field in six avoided crossings arranged by pairs of the initial states. The bottom diagrams of Fig. $1 \mathrm{~b}$ represent the different two-body FRET in a two-particle picture, omitting the third inactive atom in the three-particle picture of Fig. 1a.

In caesium these resonances can be explored for all $n<42$ using the Förster defect electric field dependence ${ }^{36}$. For each resonance, we consider the angle-averaged dipole-dipole interaction coupling term $V_{\text {dip }}$ which is proportionnal to the two transition dipole moments $\mu_{p s}$ and $\mu_{p s^{\prime}}$ between $p$ and $s$ (resp. $s^{\prime}$ ) and inversely proportional to the cube of the interatomic distance $R$ such that $V_{\text {dip }} \propto \frac{\mu_{p s} \times \mu_{p s^{\prime}}}{R^{3}}$. The $n^{2}$ dependence of the dipole moments in Rydberg atoms implies thus a $n^{4}$ dependence of the dipole-dipole interaction.

Three-body resonant interaction. Considering now a third atom, we introduce two non trivial novel FRET processes starting from $p$ or $p^{\prime}$ states as described by the following equations:

$$
\begin{aligned}
& 3 \times p \leftrightarrow s+s^{\prime}+p^{\prime} \\
& 3 \times p^{\prime} \leftrightarrow s+s^{\prime}+p
\end{aligned}
$$

Both processes are three-body coherent FRET processes and correspond to the two outer avoided crossings, (I) from $p$ and $\left(\mathrm{I}^{\prime}\right)$ from $p^{\prime}$ in Fig. 1a. They appear at specific electric fields well separated from the two-body FRET due to the finite energy $\delta_{p p^{\prime}}$. The three-body resonance conditions are respectively $\Delta_{p}=-\delta_{p p^{\prime}} / 2$ and $\Delta_{p^{\prime}}=\delta_{p p^{\prime}} / 2$. In the above three-body processes, one particle changes only weakly its energy and can be seen as assisting the two-body FRET at a different resonance 
a

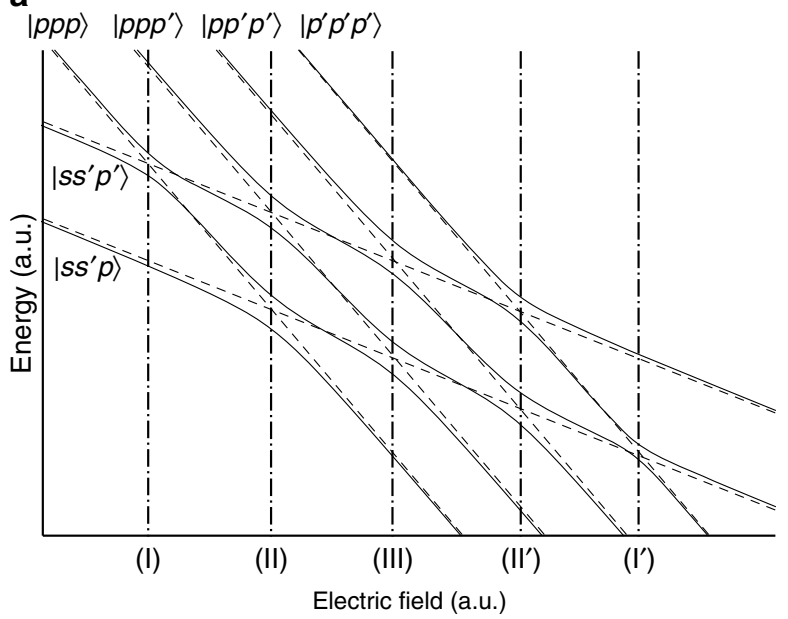

b

(I)

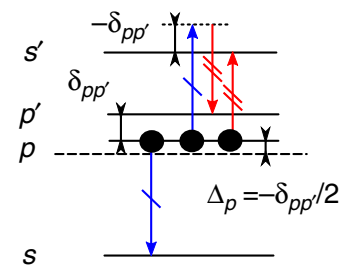

d

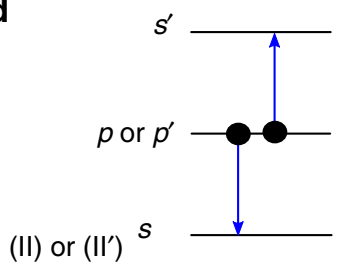

C

$\left(I^{\prime}\right)$

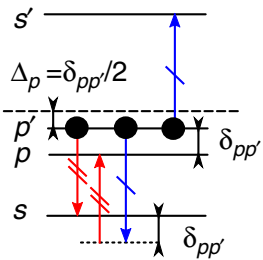

(III)

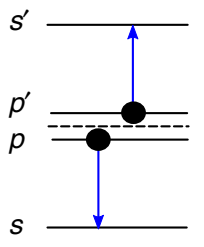

Figure 1 | Two-body and three-body FRET processes. (a) Schematic energy diagram as a function of the applied electric field for the different three-atom states involved: the dotted lines represent the energy of each state in the abscence of interactions, for example, for atoms far apart. Interactions turn the different resonances into avoided crossings marked with dash-dotted lines. They correspond to two-body resonances ((II), (III) and $\left(\mathrm{II}^{\prime}\right)$ ) and three-body resonances $\left((\mathrm{I})\right.$ and $\left.\left(\mathrm{I}^{\prime}\right)\right)$ where the resonant energy transfers detailed in (b) to (e) can take place. (d) Describes how the two-body FRET (II) (resp. (II')) from the $p$ (resp. $p^{\prime}$ ) state occurs through the exchange of a single virtual photon symbolized with two blue arrows. (e) Describes the similar two-body FRET from a $p$ and $p^{\prime}$ mixture. (b) (resp. (c)) Describes how the three-body FRET transfers the starting ppp (resp. $p^{\prime} p^{\prime} p^{\prime}$ ) state to the end state through the exchange of two different virtual photons. The first virtual photon, symbolized with blue arrows crossed with a single mark, leads the system to a virtual intermediate state before the second, symbolized with red arrows crossed with two marks, leads it resonantly to the end state. For reference, an horizontal dashed line at the energy $\left(E_{s}+E_{s^{\prime}}\right) / 2$ represents when possible the two-body resonance condition.

condition. This atom will relay the energy between the two other atoms.

The top diagrams of Fig. $1 \mathrm{~b}$ represent the three-body (I) (resp. $\left(\mathrm{I}^{\prime}\right)$ ) FRET where the centre atom, transferred to $p^{\prime}$ (resp. $p$ ), acts as the relay contributing twice to the whole energy transfer. In this figure this is displayed by the two arrows acting on the relay atom. The specificity of these processes resides in the absence of a real intermediate state, which is detuned by $\delta_{p p^{\prime}}$. Despite the use of a relay, the transfer occurs in a single step, implying a Borromean character of the relay atom which absorbs

the energy of the finite Förster defect. Indeed at the three-body resonant field the two-body FRET is forbidden and only the single three-body resonance is allowed. The overall process corresponds to the transfer of one excitation over the three atoms. When $\delta_{p p^{\prime}}$ is large compared to the dipole-dipole interactions, the three-body coupling can be perturbatively evaluated to a coherent interaction with the coupling $V_{3 \mathrm{~b}}=V_{\mathrm{dip}_{1}} V_{\mathrm{dip}_{2}} / \delta_{p p^{\prime}}$ where $V_{\mathrm{dip}_{i}}$ is the dipole-dipole interaction coupling the Borromean atom to the $i$ th other atom.

Experimental protocol summary. In our experimental set-up described in ref. 34 , the preparation in an $n P_{3 / 2}$ Rydberg state (with $n$ between 28 and 35) is produced through a three-photon resonant excitation using two intermediate states $6 P_{3 / 2}$ and $7 S_{1 / 2}$. The short lifetime of these intermediate states of around 30 and $50 \mathrm{~ns}$, together with the duration of the excitation pulse of typically $200 \mathrm{~ns}$, broadens the excitation linewidth to around $10 \mathrm{MHz}$. It limits strongly the excitation blockade, leading to high Rydberg atom densities with random distributions. The random interatomic distances and orientations in the Rydberg sample justify the use of the angle-averaged interaction at the average distance in our calculations and will prevent the observation of coherence properties. We then let the system evolve for times $\tau_{\text {delay }}$ ranging from $300 \mathrm{~ns}$ to $1 \mu \mathrm{s}$ during which the energy transfer takes place towards a final population in $s$ and $s^{\prime}$ states. Finally we apply an ionization ramp to obtain a time-of-flight (TOF) signal and extract the populations $N_{j}$ in each Rydberg state $j$, appearing in the TOF in the order $N_{s^{\prime}}, N_{p}, N_{p^{\prime}}$ and finally $N_{s}$. We will usually express the results in terms of the population transfer ratio $R_{s s^{\prime}}=\left(N_{s}+N_{s^{\prime}}\right) / \sum_{j} N_{j}$ describing the fraction of Rydberg atoms transfered to the $s$ or $s^{\prime}$ states. The population detection presents cross-talks on the order of $10-20 \%$ due to avoided crossings in the ionization path but we correct for them using a simple matrix algorithm already presented in ref. 34 . This correction requires taking a reference TOF for each considered state at a reference electric field $F_{0}$ away from any resonances. The final uncertainty in population transfer ratios $R_{s s^{\prime}}$ is evaluated to be around $\pm 0.5 \%$ for data at electric fields on the same side of the main two-body FRET resonance than $F_{0}$. On the other side, larger residual cross-talk errors up to $5 \%$ for $n=35$ can persist due to the interactions during the ionization ramp. We therefore always paid attention to take the reference TOF on the same side as the expected three-body FRET. After the initial $p$ or $p^{\prime}$ preparation, the tuning of the electric field gives access to resonances (I) and (II) (resp. $\left(\mathrm{I}^{\prime}\right)$ and $\left(\mathrm{II}^{\prime}\right)$ ), but never to resonance (III) which requires a $p$ and $p^{\prime}$ mixture.

Resonances. Figure 2 reports the results for $n=35$, which leads to the largest measured transfer due to the $n^{4}$ dependence of $V_{\text {dip }}$ and to the smallest $\delta_{p p^{\prime}}$.

In Fig. 2a, we plot the population transfer ratio versus the applied electric field $F$ and display the result from the $p$ state for different transfer delays $\tau_{\text {delay }}$. We resolve two peaks corresponding to the two-body (II) and three-body (I) FRET at $3.30 \mathrm{~V} \mathrm{~cm}^{-1}$ and $3.17 \mathrm{~V} \mathrm{~cm}^{-1}$, respectively. They are in good agreement with the values of $3.28 \mathrm{~V} \mathrm{~cm}^{-1}$ and $3.15 \mathrm{~V} \mathrm{~cm}^{-1}$, calculated using the numerical method proposed in ref. 37 , with our uncertainty on the applied electric field of $\pm 0.02 \mathrm{~V} \mathrm{~cm}^{-1}$ and on the calculated resonance position of $\pm 0.05 \mathrm{~V} \mathrm{~cm}^{-1}$. The observed two-body transfer saturation limit of $50 \%$ is due to the random distribution of Rydberg atoms in our sample leading to a statistical average between the two-body states $|p p\rangle$ and $\left|s s^{\prime}\right\rangle$. We observe an efficient three-body FRET transfer of up to $\sim 21 \%$ close to, but below, the expected maximum of $33 \%$ for a statistical average between $|p p p\rangle$ and $\left|s s^{\prime} p^{\prime}\right\rangle$. 
a

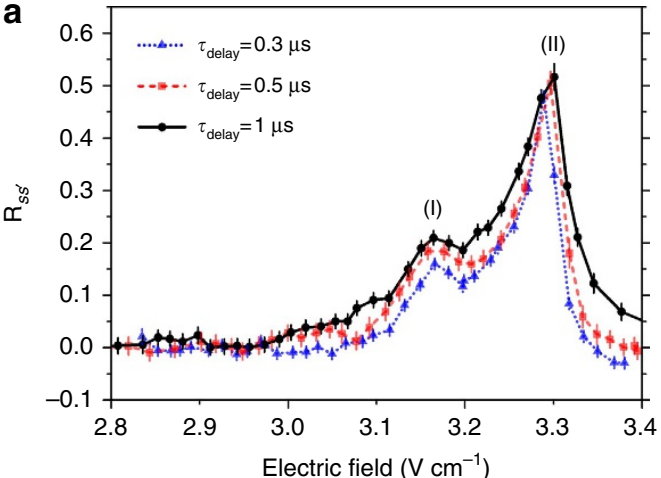

b

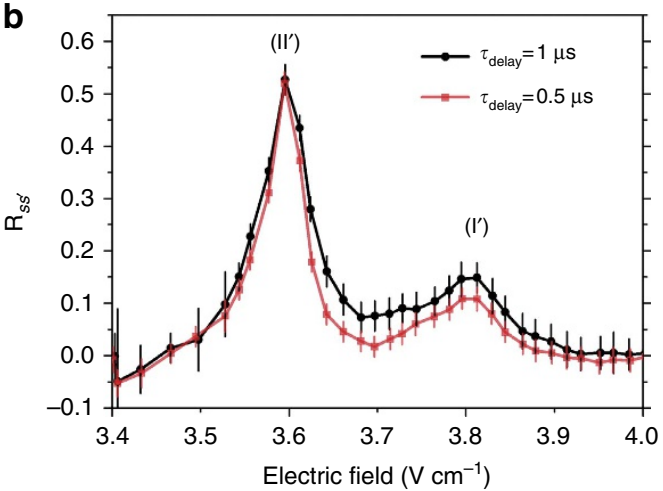

Figure 2 | Population transfer. Averaged transfer ratio $R_{s s^{\prime}}$ from the initial $p$ state (a) or $p^{\prime}$ state (b) to $s+s^{\prime}$ states for $n=35$ at different $\tau_{\text {delay }}$ versus the electric field. $\ln (\mathbf{a})$, the resonance at around $3.3 \mathrm{~V} \mathrm{~cm}^{-1}$ is attributed to the two-body FRET (II) while the resonance at around $3.17 \mathrm{Vcm}^{-1}$ is attributed to the three-body FRET (I). In (b), the resonances are attributed to $\left(\mathrm{II}^{\prime}\right)$ and $\left(\mathrm{I}^{\prime}\right)$. Each point corresponds to the average of 50-150 individual measurements. The error bars correspond to the sum of the s. e. $\mathrm{m}$. and the estimated error in the state discrimination.

Figure $2 \mathrm{~b}$ presents the analogous results from the initial $p^{\prime}$ state, corresponding to the three-body FRET of equation (3). The resonance fields are again in excellent agreement with the predicted values of $3.60 \mathrm{~V} \mathrm{~cm}^{-1}$ and $3.80 \mathrm{~V} \mathrm{~cm}^{-1}$ for (II') and (I'), respectively. In addition, we see that the two peaks are better resolved due to the increase of the energy splitting $\delta_{p p^{\prime}}$ with the electric field. It also implies a smaller three-body coupling $V_{3 \mathrm{~b}}$ and the observed transfer efficiency is smaller with around $15 \%$ transfer.

The application of different delays in Fig. 2 enables us to observe the transfer time evolution. At our experimental density of $\sim 5 \times 10^{9}$ atoms $\mathrm{cm}^{-3}$, the typical two-body FRET coupling $V_{\text {dip }}$ is expected to reach a maximum of $15 \mathrm{MHz}$ at the average interatomic distance of $3 \mu \mathrm{m}$ and the transfer is fully saturated at the shortest delays. This interaction level, larger than the excitation linewidth, is large enough to induce a partial excitation blockade effect and could induce the direct excitation of one of the two-body exciton states $\left(|p p\rangle \pm\left|s s^{\prime}\right\rangle\right) / \sqrt{2}$ still corresponding to the $50 \%$ expected saturation transfer. On the contrary, with $\delta_{p p^{\prime}} \sim 70 \mathrm{MHz}$ for FRET (I) (resp. $\delta_{p p^{\prime}} \sim 95 \mathrm{MHz}$ for FRET $\left.\left(I^{\prime}\right)\right)$, the three-body interaction coupling $V_{3 \mathrm{~b}}$ is expected around $3 \mathrm{MHz}$ (resp. $2 \mathrm{MHz}$ ) at $3-\mu \mathrm{m}$ interatomic distances. As a consequence, we observe a time evolution at the shortest delays. Unfortunately, the strong dependence of the three-body interaction $V_{3 \mathrm{~b}}$ with the interatomic distances and the random distribution of the Rydberg atoms in our sample prevent us from observing any oscillations in the energy transfer.

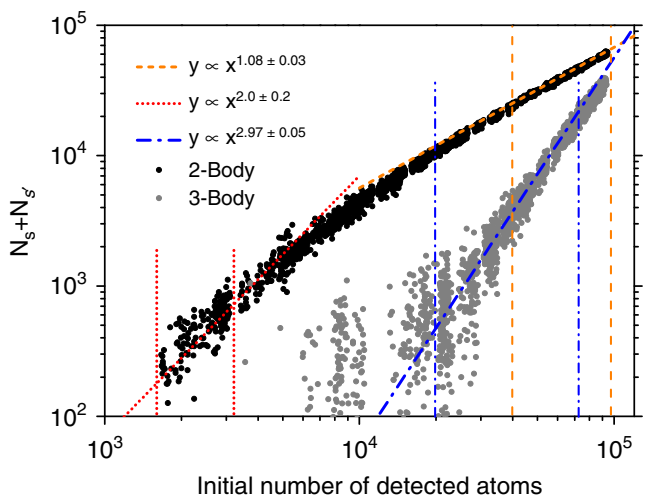

Figure 3 | Density dependence. Sum of detected atoms in $s$ and $s^{\prime}$ states, $N_{s}+N_{s^{\prime}}$ versus the initial $n=35 p^{\prime}$ Rydberg atoms density for a transfer time of $\tau_{\text {delay }}=0.5 \mu \mathrm{s}$ at a fixed electric field of $3.60 \mathrm{~V} \mathrm{~cm}^{-1}$ for the two-body FRET (black dots) in bi-logarithmic scale. Two fits check the quadratic behaviour in the low density regime (red dotted line) and the linear saturation in the high-density regime (orange dashed line). A second set of data are taken at $3.80 \mathrm{~V} \mathrm{~cm}^{-1}$ for the three-body FRET (grey dots) and a fit checks the expected cubic behaviour (blue dotted dashed line).

The result and the standard error of each fit is presented in the legend. The data range used for each fit is demarcated by couples of vertical lines with corresponding colour and style.

Density dependence. To further demonstrate the three-body character of this phenomenon, we studied the transfer dependence on the initial Rydberg atom density using the technique developed in ref. 34 . Indeed in the low density limit, a N-body process will depend as the $\mathrm{N}$-power of the initial density. We have chosen the $p^{\prime}$ starting state and a transfer time of $\tau_{\text {delay }}=0.5 \mu \mathrm{s}$ where the resonances are better resolved and the signal on one resonance is less impacted by the other resonance.

We vary the Rydberg atom density changing the duration of the Rydberg excitation pulse. Figure 3 presents in a bi-logarithmic scale the resulting number of transferred atoms as a function of the initial number of Rydberg atoms for the two-body FRET at $3.60 \mathrm{~V} \mathrm{~cm}^{-1}$ and for the three-body FRET at $3.80 \mathrm{~V} \mathrm{~cm}^{-1}$. We observe the expected quadratic behaviour of the two-body FRET at the lowest densities but also a fast saturation at moderate densities with a linear dependence once the $50 \%$ transfer limit is reached. This saturation occurs at a density about 10 times smaller than our maximum density, that is, $\sim 5 \times 10^{8}$ atoms $\mathrm{cm}^{-3}$ where the coupling $V_{\text {dip }}$ is around $1.5 \mathrm{MHz}$ at $3-\mu \mathrm{m}$ distance. This is consistent with the used delay of $\tau_{\text {delay }}=500 \mathrm{~ns}$. Moreover, the excitation blockade is negligible and cannot explain the saturation at this small density. The measured cubic dependence of the transferred population on the density for the three-body FRET signals confirms our identification of the process. For these measurements with an estimated interaction strength around $3 \mathrm{MHz}$ at the highest density, we observe the beginning of a saturation when reaching the transfer percentage of $\sim 25 \%$, close to the expected maximum of $33 \%$ in this case.

A general scheme. It is important to note here that the idea to use a non-resonant relay atom to compensate for a finite Förster defect is valid for any principal quantum number. For confirmation, we have explored the two-body and three-body FRET's for the $n=32$ Rydberg state at $F \sim 6.9 \mathrm{~V} \mathrm{~cm}^{-1}$ and $F \sim 6.63 \mathrm{~V} \mathrm{~cm}^{-1}$, respectively, as displayed in Fig. 4 . The threebody transfer ratio is about $12 \%$ and we have observed a transfer for $n=28$ of about $4 \%$. This rapid decrease of the transfer efficiency is consistent with the $V_{\text {dip }}$ strong dependence with $n$. These results confirm the general application of few-body FRET 


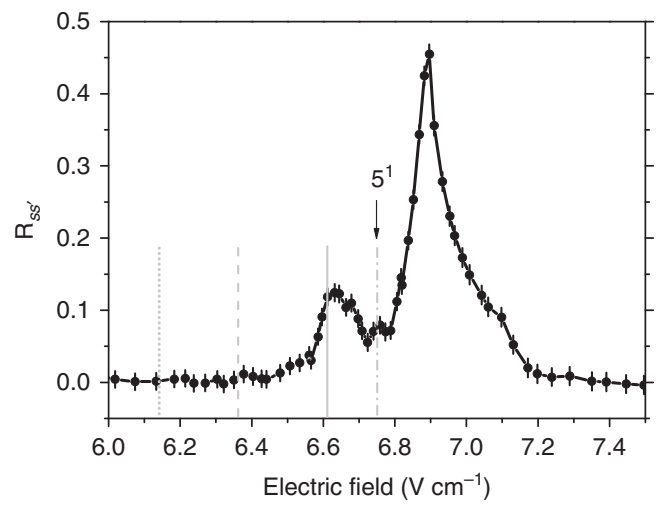

Figure 4 | Population transfer from $\boldsymbol{n}=32$. Averaged transfer ratio $R_{s s^{\prime}}$ from the initial $p$ state to $s+s^{\prime}$ for $n=32$ with a transfer time of $\tau_{\text {delay }}=1 \mu \mathrm{s}$. The solid line at $6.61 \mathrm{~V} \mathrm{~cm}^{-1}$ corresponds to the expected field for the three-body FRET. The dashed line at $6.36 \mathrm{~V} \mathrm{~cm}^{-1}$ marks the resonance electric field of the $4^{2}$ four-body FRET where two relay atoms allow the energy exchange between the last two atoms. The dotted line at $6.14 \mathrm{~V} \mathrm{~cm}^{-1}$ corresponds to the $5^{3}$ five-body FRET allowed through three relay atoms. Finally, the dashed-dotted line at $6.74 \mathrm{~V} \mathrm{~cm}^{-1}$ corresponds to the $5^{1}$ five-body FRET where a single relay atom is enough to allow the energy exchange of two pairs of atoms. Each point corresponds to the average of 150-300 individual measurements. The error bars correspond to the sum of the s. e. m. and the estimated error in the state discrimination.

processes to any atom, molecule or quantum dot, provided that the relay is able to absorb a small energy mismatch through multiple quasi-degenerate levels or a broad transition line. In Rydberg atoms, it corresponds to any resonance starting from a state with a total angular momentum $J>1 / 2$.

Our findings also confirm the validity of the many-body diffusion model introduced in ref. 38 to interpret the efficient coupling $|p p p\rangle \leftrightarrow\left|s p s^{\prime}\right\rangle$ observed through the broadening of the FRET resonance line. That model implies a multistep evolution of the Rydberg excitation transfer, as produced by strong few-body couplings as $|p p p\rangle \leftrightarrow\left|s s^{\prime} p\right\rangle \leftrightarrow\left|s p s^{\prime}\right\rangle$ where the $s$ or $s^{\prime}$ states keep exchanging their energy with surrounding $p$ atoms.

\section{Discussion}

In the following discussions, for the sake of simplicity, we will always assume a $p$ starting state and the use of the corresponding three-body or few-body resonance electric field. But of course the symmetric situation with the $p^{\prime}$ state is equivalent.

As a perspective, we can generalize the few-body FRET further to many-body (Brunnian ${ }^{39}$ ) FRET:

$$
(2 N+M) p \leftrightarrow N s+N s^{\prime}+M p^{\prime}
$$

with a resonance condition on the Förster defect defined as:

$$
\Delta_{p}=-\frac{M}{2 N} \delta_{p p^{\prime}}
$$

Within this new representation, each resonance can be labelled using the total number of involved atoms $2 N+M$ and the number of relay atoms $M$, leading to the label $(2 N+M)^{M}$. The two-body FRET is thus labelled $2^{0}$ and the 3 -body FRET $3^{1}$. It is then possible to consider the non trivial 4-body FRET $4^{2}$, the two 5 -body FRET $5^{3}$ and $5^{1}$, etc... Fig. 4 highlights with dashed lines the expected resonance fields for $4^{2}$ at $F=6.36 \mathrm{~V} \mathrm{~cm}^{-1}, 5^{3}$ at $F=6.14 \mathrm{~V} \mathrm{~cm}^{-1}$ and $5^{1}$ at $F=6.74 \mathrm{~V} \mathrm{~cm}^{-1}$. Notice that this last resonance with a very small detuning could contribute to our signal with a peak barely visible out of the noise, although no additional confirmation could be gathered: From equation (5) we find a detuning of $\delta / 4$ for the $5^{1}$ resonance, much smaller than the detuning $\delta$ for the $4^{2}$ resonance. This could in principle explain why we might observe the first and not the second. Indeed when calculating the interaction strength of these two processes we find $11 \mathrm{kHz}$ for $4^{2}$ and $25 \mathrm{kHz}$ for $5^{1}$ at $3 \mu \mathrm{m}$. But this last interaction is still too weak to explain the appearence of this peak given the used delay. The clear experimental observation and study of these resonances is a challenge for the next future, demanding improvements in the excitation and detection method, but promising new investigations on the transition from few to many-body physics ${ }^{38,40}$.

According to the theoretical study $\mathrm{in}^{33}$, the $|p p p\rangle$ state should present trimer states regardless of the chosen electric field. One can wonder how the additional few-body interactions presented here will modify these trimer states in term of accessibility and stability.

Another prospect of tunable three-body FRET could be found in quantum optics and quantum computing ${ }^{11}$ with the implementation of entanglement or few-body quantum gates. Although the randomness of the Rydberg excitations in our experiment prevented us to demonstrate the coherent nature of the observed three-body FRET, other experiments like the one in ref. 22 realized a precise control of atomic positions. That control should allow interesting applications, as discussed in the following:

The asymmetric role of the relay atom, necessarily ending in the $p^{\prime}$ state, forbids a priori a three-atom entanglement. But one can in principle symmetrize the problem using a specific atomic arrangement and electric field orientation that garanties equal dipole-dipole interactions between each atom pair. A trivial solution is to use an equilateral triangle with a perpendicular electric field. This idea should still work with 4 atoms, adding the last one out-of-plane at the proper distance giving equal coupling strengths. The implementation of a more general $\mathrm{N}$-body entanglement, for $\mathrm{N}$ larger than 4 , is much harder to assert without further investigations.

The entanglement of just two particles is already useful in many schemes and the a priori knowledge whether the entanglement has been achieved, often called heralded entanglement, is a common way to improve quantum information protocols. Our three-body FRET could provide such an heralded entanglement using the relay atom to report the entanglement of the two others. One can for instance wait for the FRET to take place and ionize the final $p^{\prime}$ atom after its transfer to a higher state via a resonant microwave field. The detection of this ion will indeed be a proof that the remaining atoms are in the entangled state $\left|\psi_{\text {heralded }}\right\rangle=\left(\left|s s^{\prime}\right\rangle+\left|s^{\prime} s\right\rangle\right) / \sqrt{2}$.

The ability to implement few-body quantum gates in a single step using few-body interactions might help reducing implementation errors when such gates are required. Evaluating accurately the feasibility and the final error of such gates is beyond the scope of this study but we will present simply one possible scheme. In a linear configuration, mapping the first and last atoms of the chain to the $s$ and $s^{\prime}$ states and the centre atom to either the $p$ or $p^{\prime}$ state will implement a controlled Swap gate, also called Fredkin gate. Indeed, if the resonance condition of equation (2) is satisfied, a $p^{\prime}$ state on the centre atom will lead to the coupling $\left|s p^{\prime} s^{\prime}\right\rangle \leftrightarrow|p p p\rangle \leftrightarrow\left|s^{\prime} p^{\prime} s\right\rangle$ where the $s$ and $s^{\prime}$ states swap. On the contrary, there is no coupling with a centre $p$ atom and the swap doesn't take place.

The resonant energy transfers we have predicted and observed pave the way towards further investigations of the frontier between few-body physics and many-body physics. Their general character, demonstrated in our work, is promising for applications as advanced control techniques in quantum physics. 


\section{Methods}

Rydberg atom source. Our experimental set-up is similar to the one described in ref. 34. We create a caesium magneto-optical trap (MOT) with a typical atomic density of several $10^{10}$ atoms $\mathrm{cm}^{-3}$ and a temperature of $\sim 100 \mu \mathrm{K}$. We then use a three-photon excitation scheme to the Rydberg states $p$ or $p^{\prime}$. The first photon is provided by the MOT cooling laser, coupling the $6 S_{1 / 2}$ ground state to the $6 P_{3 / 2}$ state at $\lambda=852 \mathrm{~nm}$. The second step couples the $6 P_{3 / 2}$ to the $7 S_{1 / 2}$ state with a diode laser at $\lambda=1470 \mathrm{~nm}$. It is switched on for $1 \mu$ s with an acousto-optic modulator. The last excitation step couples the $7 S_{1 / 2}$ state to the desired Rydberg state with a $\mathrm{cw}$ Ti:Sapphire ring laser at $\lambda=785 \mathrm{~nm}$. It is switched on for a maximum of $200 \mathrm{~ns}$ using another acousto-optic modulator within the previous laser excitation pulse. This duration limit as been chosen to ensure the absence of excitation blockade in the sample, except when the applied electric field is at the two-body resonance. The last two lasers are focused and crossed within the MOT to define a small Rydberg excitation volume of $\sim 300-\mu \mathrm{m}$ diameter. With this excitation scheme, we excite up to around $10^{5}$ Rydberg atoms with a typical Rydberg atom density of $\sim 5 \times 10^{9}$ atoms $\mathrm{cm}^{-3}$.

Electric field control and Rydberg atom detection. The MOT is located at the centre of four parallel $60 \mathrm{~mm}$ by $130-\mathrm{mm}$ wire mesh grids. The centre pair is spaced by $1.88 \pm 0.02 \mathrm{~cm}$, while the outer grids are $1.5 \mathrm{~cm}$ far from the inner grids. One centre grid is used to apply the required resonance field of several $\mathrm{V} \mathrm{cm}^{-1}$. The centre grid spacing has been measured observing the $p$ state Stark shift as a function of the applied voltage. For the final Rydberg detection, an ionizing field ramp is applied on the other centre grid with an experimental delay $\tau_{\text {delay }}$ $(300 \mathrm{~ns}-1 \mu \mathrm{s})$ after the end of the Ti:Sa excitation pulse. We apply a voltage of $900 \mathrm{~V}$ to ionize the $n=35 p$ state and $1,400 \mathrm{~V}$ to ionize $n=32$. The ions then travel $210 \mathrm{~mm}$ to reach a micro-channel plate recording the TOF signal. The different Rydberg states ionize at different fields, thus at different times within the ionization ramp. The ionization field is chosen specifically for each $n$ to optimally separate the micro-channel plate signal of $s$ and $s^{\prime}$ from $p+p^{\prime}$ on the TOF signal. This experimental cycle is then repeated with a $10-\mathrm{Hz}$ repetition rate to accumulate data.

Data analysis and selection procedure. This detection scheme provides a specific TOF signal for each Rydberg states, but the signals partially overlap and the lowest energy state $s$ only partially ionizes. To evaluate accurately the number of atoms in each state, we first measure the TOF signal of the $p, p^{\prime}, s$ and $s^{\prime}$ Rydberg states at a non-resonant electric field to serve as TOF references. The reference signals have been measured at an external electric field of $2.70 \mathrm{~V} \mathrm{~cm}^{-1}$ for Fig. $2 \mathrm{a}$ and of $4.54 \mathrm{~V} \mathrm{~cm}^{-1}$ for Figs $2 \mathrm{~b}$ and 3 . These TOF references allow us to define optimum time-integration gates and quantify the cross-talks between the various Rydberg signal to account for them ${ }^{34}$. We also measure the ionization fraction of the $s$ state and apply a correction factor on this state before processing the transfer ratio. We verify that this correction leads to an equal transfer of $s$ and $s^{\prime}$ state on the two-body FRET as expected. The residual cross-talk uncertainty is evaluated to $\pm 0.5 \%$ maximum between the following channels: $s, s^{\prime}$ and $p+p^{\prime}$. In our analysis, we choose not to discern between $p$ and $p^{\prime}$ since the two signals are too much overlapped and the residual cross-talk after correction was still on the order of $\pm 5 \%$ or more between them. Each reference and each point of the different Fig. 2 corresponds to an average of 50-150 data points selected from 400 to 500 measurements at each value of the electric field. In the case of Fig. 4, we increase the number of measurements up to 900 to save up to 300 data points. This selection is necessary as the Rydberg excitation laser is not frequency locked. The selection is automated in two steps: it first keeps data within $\pm 3 \mathrm{MHz}$ of the optimum excitation frequency while we observe an excitation linewidth of $\pm 5 \mathrm{MHz}$. It then selects an interval of $\pm 5 \%$ in Rydberg atom number containing the largest possible data set. A single interval is used for each of Figs 2 and 4 and an interval is chosen for each excitation pulse duration in Fig. 3. This avoids non-linear interaction effects to play a role within a single point in the figures. After averaging $N$ points, the statistical error of the mean (s. e. m.) is estimated by the usual formula of the measured s.d. $\sigma$ divided by $\sqrt{N}$. The error bars in Figs 2 and 4 correspond to the sum of the s.e.m. and the $\pm 0.5 \%$ residual cross-talk error. In Fig. 3 , we directly plot the whole selected data set for each used value of the Rydberg excitation pulse duration.

\section{References}

1. Buluta, I. \& Nori, F. Quantum simulators. Science 326, 108-111 (2009).

2. Bloch, I., Dalibard, J. \& Nascimbene, S. Quantum simulations with ultracold quantum gases. Nat. Phys. 8, 267-276 (2012).

3. Greiner, M., Mandel, O., Esslinger, T., Hänsch, T. W. \& Bloch, I. Quantum phase transition from a superuid to a Mott insulator in a gas of ultracold atoms. Nature 415, 39-44 (2002).

4. Bourdel, T. et al. Experimental study of the BEC-BCS crossover region in lithium 6. Phys. Rev. Lett. 93, 050401 (2004).

5. Paredes, B. et al. Tonks-Girardeau gas of ultracold atoms in an optical lattice. Nature 429, 277-281 (2004).

6. Kinoshita, T., Wenger, T. \& Weiss, D. S. Observation of a one-dimensional Tonks-Girardeau gas. Science 305, 1125-1128 (2004).
7. Kondov, S. S., McGehee, W. R., Zirbel, J. J. \& DeMarco, B. Three-dimensional Anderson localization of ultracold matter. Science 334, 66-68 (2011).

8. Jendrzejewski, F. et al. Three-dimensional localization of ultracold atoms in an optical disordered potential. Nat. Phys. 8, 398-403 (2012).

9. Weimer, H., Müller, M., Lesanovsky, I., Zoller, P. \& Büchler, H. P. A Rydberg quantum simulator. Nat. Phys. 6, 382-388 (2010).

10. Monroe, C. Quantum information processing with atoms and photons. Nature 416, 238-246 (2002).

11. Saffman, M., Walker, T. G. \& Mölmer, K. Quantum information with Rydberg atoms. Rev. Mod. Phys. 82, 2313-2363 (2010).

12. Comparat, D. \& Pillet, P. Dipole blockade in a cold Rydberg atomic sample. J. Opt. Soc. Am. B 27, A208-A232 (2010).

13. Urban, E. et al. Observation of Rydberg blockade between two atoms. Nat. Phys. 5, 110-114 (2009).

14. Gaëtan, A. et al. Observation of collective excitation of two individual atoms in the Rydberg blockade regime. Nat. Phys. 5, 115-118 (2009).

15. Wilk, T. et al. Entanglement of two individual neutral atoms using Rydberg blockade. Phys. Rev. Lett. 104, 010502 (2010).

16. Isenhower, L. et al. Demonstration of a neutral atom controlled-NOT quantum gate. Phys. Rev. Lett. 104, 010503 (2010).

17. Nipper, J. et al. Atomic pair-state interferometer: controlling and measuring an interaction-induced phase shift in Rydberg-atom pairs. Phys. Rev. X 2, 031011 (2012).

18. Safinya, K. A., Delpech, J. F., Gounanad, F., Sandner, W. \& Gallagher, T. F. Resonant Rydberg-atom-Rydberg-atom collisions. Phys. Rev. Lett. 47, 405-408 (1981).

19. FRET-Förster Resonance Energy Transfer, From Theory to Applications (eds. Medintz, I. L. \& Hildebrandt, N.) (Wiley-VCH, 2014).

20. Förster, T. Zwischenmolekulare Energiewanderung und Fluoreszenz. Ann. Phys. 437, 55-75 (1948).

21. Perrin, F. Théorie quantique des transferts d'activation entre molécules de même espéce. Cas des solutions fluorescentes. Ann. Phys. 17, 283 (1932).

22. Ravets, S. et al. Coherent dipole-dipole coupling between two single Rydberg atoms at an electrically-tuned Förster resonance. Nat. Phys. 10, 914-917 (2014)

23. Günter, G. et al. Observing the dynamics of dipole-mediated energy transport by interaction-enhanced imaging. Science 342, 954-956 (2013).

24. Barredo, D. et al. Coherent excitation transfer in a spin chain of three Rydberg atoms. Phys. Rev. Lett. 114, 113002 (2015).

25. Collini, E. et al. Coherently wired light-harvesting in photosynthetic marine algae at ambient temperature. Nature 463, 644-647 (2010).

26. Heilemann, M. et al. Multistep energy transfer in single molecular photonic wires. J. Am. Chem. Soc. 126, 6514-6515 (2004).

27. Sternlicht, H., Nieman, G. C. \& Robinson, G. W. Triplet-Triplet Annihilation and Delayed Fluorescence in Molecular Aggregates. J. Chem. Phys. 38, 1326-1335 (1963).

28. Scholes, G. D. Long-range resonance energy transfer in molecular systems. Annu. Rev. Phys. Chem. 54, 57-87 (2003).

29. Kraemer, T. et al. Evidence for Efimov quantum states in an ultracold gas of caesium atoms. Nature 440, 315-318 (2006).

30. Pollack, S. E., Dries, D. \& Hulet, R. G. Universality in three- and four-body bound states of ultracold atoms. Science 326, 1683-1685 (2009).

31. Esry, B. D., Greene, C. H. \& Burke, J. P. Recombination of three atoms in the ultracold limit. Phys. Rev. Lett. 83, 1751-1754 (1999).

32. Bendkowsky, V. et al. Rydberg trimers and excited dimers bound by internal quantum reflection. Phys. Rev. Lett. 105, 163201 (2010).

33. Kiffner, M., Li, W. \& Jaksch, D. Three-body bound states in dipole-dipole interacting rydberg atoms. Phys. Rev. Lett. 111, 233003 (2013).

34. Gurian, J. H. et al. Observation of a resonant four-body interaction in cold cesium Rydberg atoms. Phys. Rev. Lett. 108, 023005 (2012).

35. Petrov, D. S. Three-body interacting bosons in free space. Phys. Rev. Lett. 112 103201 (2014).

36. Viteau, M. et al. Melting a frozen Rydberg gas with an attractive potential. Phys Rev. A. 78, 040704(R) (2008)

37. Zimmerman, M. L., Littman, M. G., Kash, M. M. \& Kleppner, D. Stark structure of the Rydberg states of alkali-metal atoms. Phys. Rev. A. 20, 2251-2275 (1979).

38. Mourachko, I. et al. Many-body effects in a frozen Rydberg gas. Phys. Rev. Lett. 80, 253-256 (1998).

39. Yamashita, M. T., Fedorov, D. V. \& Jensen, A. S. Universality of Brunnian (N-body Borromean) four- and five-body systems. Phys. Rev. A. 81, 063607 (2010)

40. Anderson, W. R., Veale, J. R. \& Gallagher, T. F. Resonant dipole-dipole energy transfer in a nearly frozen Rydberg gas. Phys. Rev. Lett. 80, 249-252 (1998).

\section{Acknowledgements}

We are grateful to N. Hildebrandt and G. S. Schlau-Cohen for usefull discussions on biological terminology on FRET. This work was supported by ANR program COCORYM (ANR-12-BS04-0013), EU Marie-Curie program ITN COHERENCE FP7-PEOPLE-2010-ITN (R.F.), the public grant CYRAQS from Labex PALM (ANR-10LABX-0039) (P.C.) and the EU H2020 FET Proactive project RySQ (grant N. 640378) 


\section{Author contributions}

P.C. and P.P. conceived the experiment, R.F. and B.P. performed the experiment and A.Z. contributed to the experiment. R.F. and P.C. wrote the paper. P.C., P.P. and E.A. supervised the work.

\section{Additional information}

Competing financial interests: The authors declare no competing financial interests.

Reprints and permission information is available online at http://npg.nature.com/ reprintsandpermissions/
How to cite this article: Faoro, R. et al. Borromean three-body FRET in frozen Rydberg gases. Nat. Commun. 6:8173 doi: 10.1038/ncomms9173 (2015).

(c) (i) This work is licensed under a Creative Commons Attribution 4.0 International License. The images or other third party material in this article are included in the article's Creative Commons license, unless indicated otherwise in the credit line; if the material is not included under the Creative Commons license, users will need to obtain permission from the license holder to reproduce the material. To view a copy of this license, visit http://creativecommons.org/licenses/by/4.0/ 